\title{
CLIMA SOCIAL FAMILIAR Y RENDIMIENTO ACADÉMICO EN ALUMNOS DE SEXTO GRADO DE ALGUNAS ESCUELAS PÚBLICAS Y PRIVADAS DEL NORESTE DE MÉXICO
}

\section{FAMILY SOCIAL CLIMATE AND ACADEMIC PERFORMANCE IN SIXTH-GRADE STUDENTS OF SOME PUBLIC AND PRIVATE SCHOOLS IN NORTHEAST MEXICO}

\author{
Marta Emilia Soto Ovares \\ Universidad Adventista de Centro América \\ martac@um.edu.mx \\ https://orcid.org/0000-0003-0348-201X \\ Jaime Rodríguez Gómez \\ Instituto Universitario del Sureste, México \\ Email: jar@um.edu.mx \\ https://orcid.org/0000-0003-4761-140X
}

\begin{abstract}
RESUMEN
Esta investigación descriptiva y correlacional buscó observar cuál era la relación que existía entre el clima social familiar y el rendimiento académico en 204 niños de sexto año de algunas escuelas públicas y privadas, de un municipio del noreste de México, a quienes se les administró la Escala de Clima Social Familiar de Moos, Moos y Trickett (1989). Se recogieron datos del promedio de aprovechamiento de cada estudiante en el bimestre anterior a la administración del instrumento. Al analizar los resultados obtenidos, se encontró una relación positiva entre el clima social familiar y el rendimiento académico de los alumnos del estudio. Los resultados muestran la importancia de fomentar las relaciones y la estabilidad familiar para contribuir al desempeño escolar de los estudiantes
\end{abstract}

Palabras clave: clima social familiar, rendimiento académico

\section{ABSTRACT}

This descriptive and correlational study was designed to observe what was the relationship between the family social climate and the academic performance in 204 sixth-grade children from some public and private schools in a city in northeast Mexico. A Spanish versión of the Family Environment Scale by Moos, Moos, and Trickett (1989) was administered to the students and the average achievement of each student was collected in the two-month period prior to the administration of the instrument. When analyzing the results obtained, a positive relationship was found between the family environment and the academic performance of the participant 
students. The results show the importance of fostering relationships and family stability to contribute to the school performance of the students.

Keywords: family social environment, academic performance

\section{Introducción}

Uno de los aspectos más importantes del proceso de enseñanza-aprendizaje es el rendimiento académico del estudiante. Cuando se habla de evaluarlo y mejorarlo, se analizan los factores que pueden influir sobre él, tales como factores socioeconómicos, programas de estudio, metodología de enseñanza que utiliza el maestro y conceptos previos que tienen los alumnos (Edel Navarro, 2003).

Jiménez (citado en Edel Navarro, 2003) observó que se puede tener una buena capacidad intelectual sin que se esté obteniendo un rendimiento académico adecuado, ya que el rendimiento académico es un fenómeno que aparece debido a múltiples factores.

Diversas investigaciones han procurado encontrar explicaciones al bajo rendimiento académico de los alumnos. Ellas van desde estudios exploratorios, descriptivos y correlacionales hasta estudios explicativos (Edel Navarro, 2003). México no es la excepción y muchos estudios se vienen realizando desde hace algunos años. Los responsables en ejercer la autoridad educativa no escatiman recursos financieros para definir estrategias con el fin de mejorar la enseñanza y atender los compromisos con la Organización para la Cooperación y el Desarrollo Económicos (OCDE), un organismo internacional que promueve políticas que mejoran el bienestar económico y social de las personas alrededor del mundo (Egremy, 2010).

Se calcula que un $20 \%$ de la población infantil en México presenta bajo rendimiento escolar. Los costos econó- micos y sociales del problema son enormes, pero más importante aún es el impacto psicológico que el niño sufre, ya que puede dejar secuelas importantes en su desarrollo que afectan su calidad de vida (Roel Favela, 2003).

El proceso educativo no solamente se realiza en las aulas, sino también en el mismo seno familiar y en la sociedad. Por ello, se delimitaron dos variables que fueron objeto de estudio en esta investigación: clima social familiar y rendimiento académico.

En el campo de la investigación psicoeducativa y social, se han realizado muchos estudios que señalan que el clima y el funcionamiento socio-familiar son factores con mucha incidencia en el rendimiento académico de los niños. Se ha observado que un adecuado funcionamiento familiar hace que los niños tengan un desarrollo correcto, mayor estimulación y, por lo tanto, un desarrollo general más completo y adecuado para los miembros que lo componen (Robledo Ramón y García Sánchez, 2009).

El clima familiar es uno de los factores de mayor importancia en el ajuste psicosocial del niño y del adolescente. Está constituido por el ambiente percibido e interpretado por los miembros que integran la familia, y ha mostrado ejercer una influencia significativa tanto en la conducta, como en el desarrollo social, físico, afectivo e intelectual de los integrantes. Un clima familiar positivo hace referencia a un ambiente que se fundamenta en la cohesión afectiva entre padres e hijos, el apoyo, la confianza, la intimidad y la comunicación familiar 


\section{CLIMA SOCIAL FAMILIAR Y RENDIMIENTO ACADÉMICO}

abierta y empática. Un clima familiar negativo, por el contrario, carente de los elementos mencionados, se ha asociado con el desarrollo de problemas de comportamiento y de rendimiento académico en niños y adolescentes (Moreno Ruiz, Estévez López, Murgui Pérez y Musitu Ochoa, 2009).

Gerstenfeld (1995) señaló que el clima educacional del hogar es el elemento más predominante para que se alcancen los logros educativos de los niños y jóvenes, explicando que entre un $40 \% \mathrm{y}$ un $50 \%$ de los casos el impacto resulta de las características del contexto socioeconómico y familiar de la infraestructura de la vivienda en la que reside $\mathrm{y}$, por último, del nivel de organización que posee la familia.

La UNESCO (2000) declaró que los factores contextuales o extraescolares que influyen en el rendimiento académico de los alumnos están estrechamente ligados al entorno donde funcionan las escuelas y al lugar donde viven los alumnos.

Zavala García (2001) observó que el $53.5 \%$ de los alumnos de quinto año evaluados en su investigación, sobre sus intereses vocacionales y tipos caracterológicos, expresan que el clima familiar que los envuelve presenta una estructura inadecuada, caracterizada por la inestabilidad; mientras que ningún alumno del grupo evaluado refiere vivir un ambiente estable en su hogar, el $47.6 \%$ comunica que no se siente apoyado por los miembros de su familia al momento de tomar decisiones o actuar, mientras que el $39.2 \%$ manifiesta vivir con inadecuados niveles de comunicación.

Lozano Díaz (2003) concluye que los datos sobre la relación existente entre el fracaso escolar y los determinantes familiares revelan que el nivel educativo de los padres no es el componente familiar más influyente en el rendimiento, sino que se trata de los componentes del clima familiar; es decir, aunque una buena formación académica de los padres y un ambiente cultural positivo favorecen el rendimiento escolar, son las variables afectivas y relacionales las que se destacan como factores de apoyo o detrimento del rendimiento.

Vallejo Casarín y Mazadiego Infante (2006) hallaron que los estilos parentales de crianza están relacionados con el grado de aprovechamiento escolar de los hijos, su ajuste psicológico a la escuela y a sus compañeros, así como su motivación escolar influenciada por la familia. Observaron que los padres influyen en el rendimiento académico de los hijos desde la infancia hasta la adultez.

Otro estudio (Paz, 2007) mostró que los niños cuyas familias son disfuncionales tienen una frecuencia significativamente mayor de problemas en el desempeño escolar que aquellos con familias funcionales. Se encontró un $71 \%$ de niños con problemas en el desempeño escolar proveniente de familiar disfuncionales. En cambio, en las familias funcionales, solo un $34 \%$ de los niños presentaban problemas de rendimiento escolar.

Anabalón Mercado, Carrasco Paiva, Díaz Elgueta, Gallardo Urrutia y Cárcamo Vásquez (2008) encontraron que la participación de los padres en la educación de sus hijos trae consigo diversas ventajas. Cuando los padres participan en la educación de sus niños, se obtienen beneficios para ambos grupos, ya que frecuentemente mejora la autoestima del niño y ayuda a los padres a una mejor comprensión del proceso de enseñanza. Es más, cuando los padres participan proactivamente en la educación escolar, se producen resultados positivos, como 
mayor asistencia, disminución de la deserción, mejoramiento de las actitudes y conducta del estudiante, comunicación positiva padre-hijo y mayor apoyo de la comunidad a la escuela.

Guerra (citado en Gonzales Pajuelo y Pereda Infantes, 2009) estudió las características del clima social familiar y su relación con el rendimiento académico escolar y llegó a la conclusión de que los adolescentes que provienen de hogares bien organizados muestran una buena disposición a obtener mejores calificaciones en el colegio. El ambiente escolar que se estimula en la comunicación ejerce una influencia significativa sobre el rendimiento académico.

El estudio de las relaciones familia-escuela comenzó a suscitar interés a partir de la década comprendida entre 1950 y 1960, momento en el que las evidencias empíricas empezaron a señalar el efecto que los factores sociales, en general, tenían sobre el rendimiento educativo. Entre estos, los factores relativos al entorno familiar explicaban las diferencias de rendimiento en mayor medida que otros factores, de tal manera que los logros escolares del alumnado estaban relacionados con aspectos sociales y culturales y con experiencias de aprendizaje, actitudes y expectativas presentes en el contexto familiar (Gil Flores, 2009).

Un estudio (Salazar Flores, López Sánchez y Romero Ramírez, 2010) muestra que el ambiente familiar de los niños que cursan la escuela primaria es de suma importancia para su rendimiento académico, ya que la familia interviene en el desarrollo cognitivo, psicológico y social de sus hijos. En ocasiones, los padres mantienen una idea errónea de que la escuela es para que sus hijos sean educados por los profesores, pero dejan de lado su papel de padres, quienes también deben involucrarse en la educación de sus pequeños. Esto permitiría un trabajo en conjunto entre escuela y familia para que el niño mantenga un rendimiento académico bueno.

Al momento de buscar las causas del bajo rendimiento académico, también se apunta hacia los programas de estudio, los grupos muy grandes en las aulas, la falta de recursos de las instituciones y, raras veces, al papel de los padres y su actitud de creer que su responsabilidad acaba donde empieza la de los maestros. Este estudio intenta primeramente reflexionar y luego investigar si el clima social que se percibe en el ámbito familiar puede afectar el rendimiento académico en los alumnos.

Benites (citado en Gonzales Pajuelo y Pereda Infantes, 2009) afirmó que el clima familiar está relacionado con las interacciones que los padres desarrollan con sus hijos en el hogar; estas pueden variar en cantidad y calidad e inciden de alguna forma en el rendimiento académico de los niños. Como se sabe, el tipo de interacción familiar que establecen los sujetos desde su temprana infancia ejerce influencia en sus diferentes etapas de vida, facilitando o dificultando las relaciones en las diferentes esferas de actividad: educativa, formativa, social y familiar.

Cada persona tiene necesidades que debe satisfacer y que son muy importantes para su calidad de vida. La familia es el primer lugar en donde el niño aprende a satisfacer esas necesidades, lo que en el futuro le servirá de apoyo para integrarse a un medio y a su comunidad. Una de las funciones más importantes de la familia es, en este sentido, satisfacer las necesidades de sus miembros a nivel 


\section{CLIMA SOCIAL FAMILIAR Y RENDIMIENTO ACADÉMICO}

biológico, económico, educativo, psicológico, afectivo y social (Rosales Piña y Espinosa Salcido, 2008).

\section{La familia}

La familia representa la red básica de las relaciones sociales en su máxima expresión cultural y emocional. Además de ser la institución más compleja por su componente afectivo y por su papel en los procesos de reproducción mental y social, recibe todas las tensiones y condiciones del medio. La familia se convierte en una especie de síntesis de todas las contradicciones sociales que se reflejan en ella, más o menos fuertemente, dependiendo de sus reservas de cohesión social (Zamudio, citado en de la Torre Holguín, 2015).

Gonzales Pajuelo y Pereda Infantes (2009) conceptualizan a la familia como el conjunto de personas que viven juntas, que se relacionan unas con otras y que comparten sentimientos, responsabilidades, informaciones, costumbres, valores, mitos y creencias. Cada uno de los miembros son capaces de participar de ciertos roles que permiten que se mantenga un equilibrio en ella. Como institución social, es un sistema de fuerzas que constituyen un verdadero núcleo de apoyo para sus miembros y la comunidad en que viven.

Benites (citado en Gonzales Pajuelo y Pereda Infantes, 2009) señaló que la familia sigue siendo considerada como la estructura básica de la sociedad. Su función más importante es servir como agente socializador que permite adecuar las condiciones y experiencias vitales que facilitan el desarrollo óptimo psicosocial de los hijos.

\section{Tipos de familias}

De acuerdo con la Organización de las Naciones Unidas (citada en Gonzales Pajuelo y Pereda Infantes, 2009), existen nueve tipos de familias: (a) la familia nuclear, integrada por padres e hijos, (b) las familias uniparentales o monoparentales, que se forman tras el fallecimiento de uno de los cónyuges, el divorcio, la separación, el abandono o la decisión de no vivir juntos, (c) las familias polígamas, en las que un hombre vive con varias mujeres o, con menos frecuencia, una mujer se casa con varios hombres, (d) las familias compuestas, que habitualmente incluyen tres generaciones: abuelos, padres e hijos que viven juntos, (e) las familias extensas, que, además de tener tres generaciones, está conformada por otros parientes tales como tíos, primos o sobrinos que viven en el mismo hogar, (f) las familias reorganizadas, que viven con otros matrimonios o cohabitan con personas que tuvieron hijos con otras parejas, (g) las familias inmigrantes, compuestas por miembros que proceden de otros contextos sociales, generalmente del campo hacia la ciudad, (h) las familias apartadas, en las que existe aislamiento y distancia emocional entre sus miembros e (i) las familias enredadas, que son familias de padres predominantemente autoritarios.

\section{Funciones de la familia}

La familia posee diversos tipos de funciones. Según de la Torre Holguín (2015), entre otras están las siguientes: (a) función biológica: procrear para asegurar la continuidad de la humanidad; (b) función protectora: asegurar y cuidar a la población vulnerable; (c) función educativa: socializar a los niños en los sentimientos y los patrones de comportamiento cultural; (d) función económica: satisfacer las necesidades básicas y de recreación en los miembros de la familia; (e) función afectiva: fomentar las 
emociones que generan armonía entre los miembros de la familia, produciendo autoconfianza, autoestima y realización personal; y (f) función recreativa: proporcionar actividades de juego que permitan el descanso, la estabilidad y el equilibrio en los niños.

\section{La teoría del clima social familiar de Moos}

Moos y Trickett. Rosales Piña y Espinosa Salcido (2008) señalaron que la teoría del clima social familiar de Moos, Moos y Trickett tiene como base teórica la psicología ambientalista, la cual comprende una amplia área de investigación relacionada con los efectos psicológicos del ambiente y su influencia sobre el individuo. También afirman que esta es un área de la psicología cuyo foco de investigación es la interrelación del ambiente físico con la conducta y la experiencia humanas.

Psicología ambientalista. Holahan (1996) y Lévy-Leboyer (1985) señalan que la psicología ambientalista comprende una amplia área de investigación relacionada con los efectos psicológicos del ambiente y su influencia sobre el individuo. También afirman que es un área de la psicología cuyo foco de investigación es la interrelación del ambiente físico con la conducta y la experiencia humanas. Este énfasis en la interrelación del ambiente y la conducta es importante, porque no solamente los escenarios físicos afectan la vida de las personas: los individuos también influyen activamente sobre el ambiente.

\section{Dimensiones y áreas del clima social familiar}

De acuerdo con Mikulic y Casullo (2016), muchos autores coinciden en afirmar que la interacción de los miem- bros de la familia produce algo que ha dado en llamarse clima y que estos estudios han caracterizado algunos elementos del funcionamiento de las familias y han demostrado que existe relación entre el clima y la conducta de sus miembros.

Moos (citado en Mikulic y Casu1lo, 2016) manifiesta que, para estudiar o evaluar el clima social familiar, son tres las dimensiones o atributos afectivos que hay que tener en cuenta, para lo cual ha elaborado diversas escalas de clima social aplicables a diferentes tipos de ambiente, como es el caso de la Escala del Clima Social en la Familia (FES). Ella indaga sobre las interrelaciones que se dan entre los miembros de la familia donde se cumplen las funciones de comunicación e interacción. El desarrollo personal puede ser fomentado por la vida en común, así como también la organización y el grado de control que ejercen unos miembros sobre otros, en términos de las siguientes dimensiones: relaciones, desarrollo y estabilidad.

\section{Rendimiento académico}

López Mero, Barreto Pico, Mendoza Rodríguez y Del Salto Bello (2015) manifiestan que el rendimiento académico depende tanto del individuo como del entorno familiar donde este se desarro1la. Los estudiantes que presentan bajo rendimiento académico son muchas veces marginados, tanto dentro como fuera del salón de clases, ocasionando que adquieran baja autoestima.

Un estudio reciente (Martínez González, Inglés Saura, Piqueras Rodríguez y Ramos Linares, 2010) demostró que tanto los padres como los amigos son muy importantes para alcanzar el éxito en el rendimiento académico y laboral de un individuo, así como en su bienestar físico y emocional. 


\section{Relación entre el clima social familiar y} el rendimiento académico de los niños

Un estudio realizado por Salazar Flores et al. (2010) demostró que el ambiente familiar de los niños que cursan la escuela primaria es de suma importancia para su rendimiento académico, ya que la familia interviene en el desarrollo cognitivo, psicológico y social de sus integrantes. Los niños de este estudio se hallaban dentro de la clasificación de hijos de padres autoritarios y de padres negligentes; los primeros, porque son muy exigentes con los niños, ya que los obligan a estudiar sin recibir o compartir sus inquietudes, mientras que a los segundos no les prestan atención y no tienen participación en los eventos escolares ni en las tareas escolares, por lo que los pequeños manifiestan un nivel escolar bajo, pues no se sienten tan comprometidos con sus estudios, por diversas situaciones socioemocionales.

Un estudio muy reciente (Lastre, López y Alcázar, 2018) determinó que existe una relación muy significativa entre el apoyo familiar y el rendimiento académico en niños de educación primaria. El tiempo empleado por los padres a estudiar, orientar y explicar a sus hijos los deberes escolares, aunque no posean un grado académico, es vital especialmente si logran entender la función de servir de guías y facilitadores en el proceso de enseñanza-aprendizaje.

Otro estudio (Gonzales Pajuelo y Pereda Infantes, 2009) observó una correlación entre el clima social familiar y el rendimiento escolar, donde un $80 \%$ de los alumnos con un clima social familiar inadecuado mostraba un rendimiento escolar bajo, mientras que solo un 5\% con un clima social familiar adecuado tenía rendimiento bajo.

Un estudio reciente (Santín Gonzá- lez, 2001) demostró que determinadas características socioeconómicas, así como determinados hábitos, influyen significativamente sobre el rendimiento en la escuela. El resultado más importante es que, en el conjunto de países evaluados, el nivel de estudios de los padres afecta en gran medida en los resultados académicos de sus hijos.

Otro estudio (González Barbera, 2003) mostró que el nivel socioeducativo pobre de los padres puede influir en que los jóvenes mantengan un bajo rendimiento académico. El control que ejercen los progenitores sobre sus hijos, especialmente en periodos laborales, el tiempo que pasan estos en la calle con sus iguales y la poca asistencia de los padres a reuniones en el centro educativo son factores que afectan negativamente la actividad académica de los hijos. El estudio revela que una relación fluida entre la familia y el centro educativo favorece no solo la calidad del rendimiento de los alumnos, sino también su desarrollo integral.

\section{Diseño del estudio}

\section{Metodología}

El estudio fue descriptivo y correlacional. Se usaron las técnicas cuantitativas. La investigación midió dos variables: el clima social familiar y el rendimiento académico. La variable dependiente fue el rendimiento académico, con un nivel de medición de intervalo y valores de 5 a 10 . La variable independiente fue el clima social familiar. Fue una variable de tipo cuantitativo, representada por las calificaciones obtenidas en la Escala de Clima Social Familiar de Moos, Moos y Trickett (1989), la cual considera tres dimensiones con sus respectivas áreas o subescalas de evaluación: (a) relaciones (RR), con cohesión, expresividad y conflictos; (b) desarrollo 
(DS), con autonomía, actuación, intelectual-cultural, social-recreativo y moralidad religiosa; y (c) estabilidad (ES), con organización y control.

\section{Población}

Este estudio tuvo como población a los alumnos de sexto grado de las instituciones educativas primarias del municipio de Montemorelos, Nuevo León, México.

\section{Muestra}

La muestra fue elegida de forma aleatoria por racimos, definidos por los grupos establecidos en las escuelas. Estuvo conformada por 204 alumnos de ambos sexos de sexto grado del Instituto Soledad Acevedo de los Reyes, del Instituto Hidalgo y de las escuelas Celso Flores, Valeriano García, Mariano Escobedo, Lázaro Cárdenas y Luis de la Garza Parás.

\section{Instrumentos}

En esta investigación se administró la Escala de Clima Social Familiar, elaborada por Moos et al. (1989). Esta escala aprecia las características socioambientales de todo tipo de familias y evalúa y describe las relaciones interpersonales entre los miembros de la familia, los aspectos de desarrollo que tienen mayor importancia en ella y su estructura básica. Cuenta con 90 ítems agrupados en tres dimensiones: relaciones, desarrollo y estabilidad.

La dimensión relaciones mide el grado de comunicación y libre expresión al interior de la familia y el grado de interacción conflictiva que la caracteriza, integrada por las siguientes áreas: (a) cohesión (CO), que mide el grado en el que los miembros del grupo familiar están compenetrados y se apoyan entre sí; (b) expresividad (EX), que explora el grado en el que se permite y anima a los miembros de la familia a actuar libremente y a expresar directamente sus sentimientos; y (c) conflictos (CT), que mide el grado en el que se expresan libre y abiertamente la cólera, la agresividad y el conflicto entre los miembros de la familia.

La dimensión desarrollo evalúa la importancia que tienen dentro de la familia ciertos procesos de desarrollo personal, que pueden ser fomentados o no, por la vida en común. Esta dimensión está integrada por las siguientes áreas: (a) autonomía (AU), que es el grado en el que los miembros de la familia están seguros de sí mismos, son independientes y toman sus propias decisiones; (b) actuación (AC), que es el grado en el que las actividades (tales como el colegio o el trabajo) se enmarcan en una estructura orientada a la acción-competencia; (c) intelectual-cultural (IC), que es el grado de interés en las actividades de tipo político, intelectual, cultural y social; (d) social-recreativa (SR), que explora el grado de participación en actividades de esparcimiento; y (e) moralidad-religiosidad (MR), que mide la importancia que se le da a las prácticas y valores de tipo ético y religioso.

La dimensión estabilidad proporciona información sobre la estructura y organización de la familia y sobre el grado de control que normalmente ejercen unos miembros de la familia sobre otros. Está integrada por las siguientes áreas: (a) organización (OR), que mide la importancia que se le da en el hogar a una clara organización y estructura al planificar las actividades y responsabilidades de la familia; y (b) control (CN), que es el grado en el que la dirección de la vida familiar se atiene a reglas y procedimientos establecidos. 


\section{CLIMA SOCIAL FAMILIAR Y RENDIMIENTO ACADÉMICO}

Esta escala aprecia características socio ambientales de todo tipo de familias. Evalúa y describe las relaciones interpersonales entre los miembros de la familia, los aspectos de desarrollo que tienen mayor importancia en ella y su estructura básica para una población de 11 a 12 años y de aplicación individual. Las categorías de la escala de Likert utilizada son las siguientes: nunca, raramente, a veces, generalmente y siempre (Ver Tabla 1).

\section{Tabla 1}

\section{Dimensiones, áreas e items de la Escala de Clima Social Familiar}

\begin{tabular}{clc}
\hline Dimensión & \multicolumn{1}{c}{ Área } & \multicolumn{1}{c}{ Ítems } \\
\hline Relaciones & Cohesión & $1,11,21,31,41,51,61,71$ y 81 \\
& Expresividad & $2,12,22,32,42,52,62,72$ y 82 \\
& Conflictos & $3,13,23,33,43,53,63,73$ y 83 \\
Desarrollo & Autonomía & $4,14,24,34,44,54,64,74$ y 84 \\
& Actuación & $5,15,25,35,45,55,65,75$ y 85 \\
& Intelectual-cultural & $6,16,26,36,46,56,66,76$ y 86 \\
& Social-recreativa & $7,17,27,37,47,57,67,77$ y 87 \\
& Moralidad-religiosidad & $8,18,28,38,48,58,68,78$ y 88 \\
& Organización & $9,19,29,39,49,59,69,79$ y 89 \\
& Control & $10,20,30,40,50,60,70,80$ y 90 \\
\hline
\end{tabular}

Una de las variables más empleadas o consideradas por los docentes e investigadores para aproximarse al rendimiento académico es la de calificaciones escolares, razón por la cual existen estudios que pretenden calcular algunos índices de fiabilidad y validez de este criterio considerado como "predictivo" del rendimiento académico. El rendimiento académico es entendido como una medida de las capacidades respondientes o indicativas que manifiestan, en forma estimativa, lo que una persona ha aprendido como consecuencia de un proceso de instrucción o formación. De la misma forma, desde una perspectiva propia del estudiante, se define el rendimiento académico como la capacidad de responder satisfactoriamente frente a estímulos educativos, susceptibles de ser interpretados según objetivos o propósitos educativos preestablecidos (Edel Navarro, 2003).
El rendimiento académico de los alumnos de sexto grado de primaria del Instituto Soledad Acevedo de los Reyes, el Instituto Hidalgo y las Escuelas Celso Flores, Valeriano García, Mariano Escobedo, Lázaro Cárdenas y Luis de la Garza Parás se determinó mediante el promedio de aprovechamiento de cada estudiante en el bimestre anterior a la aplicación del instrumento elaborado para esta investigación. La escala utilizada corresponde a un rango de 5 a 10 , según lo establece la Secretaría de Educación Pública de México (normas generales para la evaluación, acreditación, promoción y certificación en la educación básica, 2013. Diario Oficial de la Federación, Artículo 8).

Toda la información recopilada se organizó en carpetas por grado a fin de asegurarse un manejo adecuado y fidedigno de los datos recolectados. 


\section{Recopilación de datos}

La recolección de datos para este estudio se realizó por medio de un cuestionario que se administró a los estudiantes. Para realizar la recolección de los datos, se visitó cada escuela y se administró la escala al grupo o grupos de sexto grado asignados por el director de la institución mediante una cita previa.

La información sobre el rendimiento académico fue suministrado por la secretaría de cada escuela a partir del registro de calificaciones.

\section{Resultados}

Los objetivos de este estudio han sido determinar la relación entre la dimensión relaciones del clima social familiar y el rendimiento escolar, la relación entre la dimensión desarrollo del clima social familiar y el rendimiento escolar y la relación entre la dimensión estabilidad del clima social familiar y el rendimiento escolar en los niños participantes.

\section{Descripción demográfica de la muestra}

En el estudio participaron $102(50 \%)$ varones y $102(50 \%)$ niñas, haciendo un total de 204 alumnos encuestados.

La edad de los participantes estuvo comprendida entre los 10 y los 13 años. La mayor cantidad de estudiantes encuestados corresponden a la edad de 11 años $(59,8 \%)$, seguidos por los de 12 años (36,8\%); luego, los de 10 años $(2 \%)$ y, por último, los de 13 años $(1,5 \%)$.

De los 204 estudiantes de la muestra, 51 procederían de dos centros educativos de control privado, el Instituto Hidalgo y el Instituto Soledad Acevedo de los Reyes y 153 de cinco centros edu- cativos de control público, las escuelas Celso Flores Zamora, Valeriano García Jiménez, Mariano Escobedo, Lázaro Cárdenas y Luis de la Garza Parás (ver Tabla 2).

La mayoría de estudiantes partitipantes convivía con sus dos padres, $(n$ $=156,76,5 \%), 42$ estudiantes $(20,6 \%)$ solo vivían con uno de ellos y solamente $4(2 \%)$ no vivían con ninguno de ellos. Dos de ellos se abstuvieron de contestar.

Tabla 2

Distribución de la muestra por centro educativo

\begin{tabular}{lrr}
\hline \multicolumn{1}{c}{ Centro educativo } & \multicolumn{1}{c}{$n$} & \multicolumn{1}{c}{$\%$} \\
\hline Celso Flores & 37 & 18.1 \\
Valeriano García & 17 & 8.3 \\
Mariano Escobedo & 50 & 24.5 \\
Lázaro Cárdenas & 25 & 12.3 \\
Instituto Hidalgo & 5 & 2.5 \\
Luis de la Garza & 24 & 11.8 \\
ISAR & 46 & 22.5 \\
Total & 204 & 100.0 \\
\hline
\end{tabular}

\section{Comportamiento de las variables del estudio}

Rendimiento escolar. En la Figura 1 se presenta el análisis de la distribución de la muestra por promedio obtenido durante un bimestre escolar. El estudio revela que, de los estudiantes encuestados, un alumno presenta un nivel reprobatorio, cinco niños $(2 \%)$ se ubican en el nivel mínimo de pase, 124 de ellos (61\%) se ubican en el nivel regular y 74 alumnos (36\%) se ubican en el nivel alto-óptimo. El porcentaje más grande lo ocupan los niños que presentan calificaciones entre 7 y $8(61 \%)$. De igual forma se observa la distribución del rendimiento académico, donde la media fue de 8.6 $(\mathrm{DE}=0.834)$. 


\section{CLIMA SOCIAL FAMILIAR Y RENDIMIENTO ACADÉMICO}

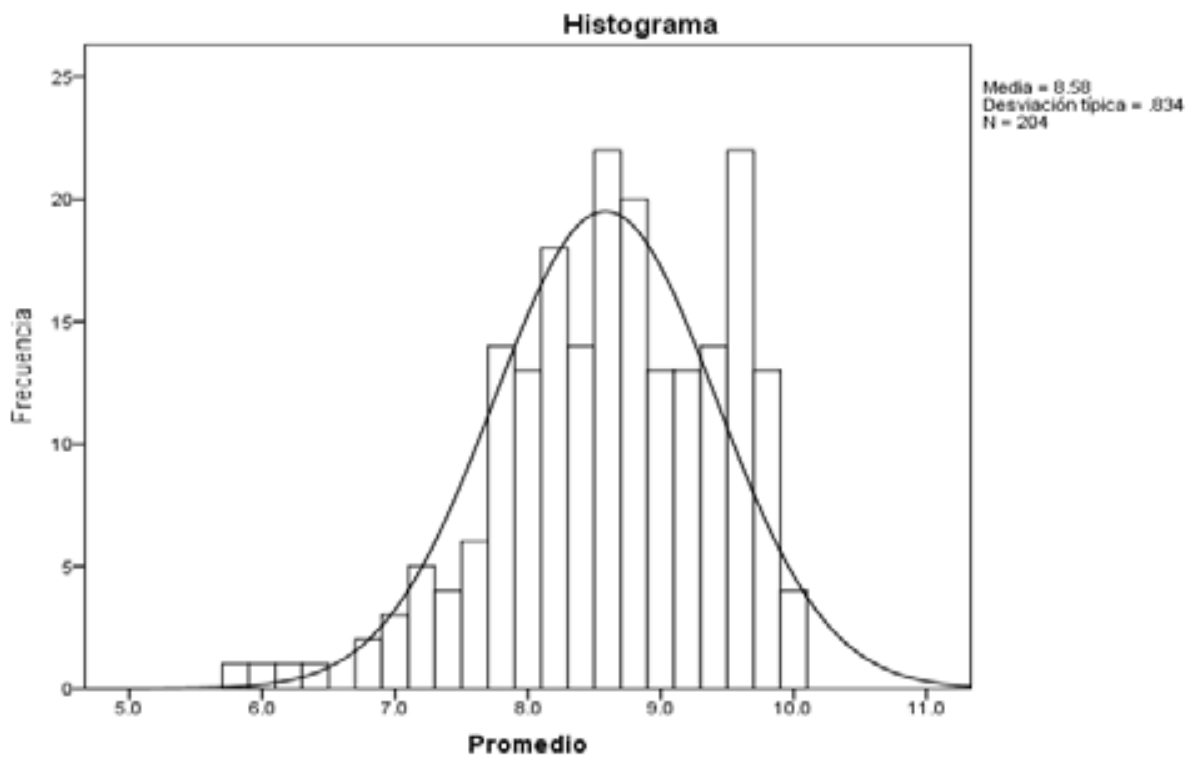

Figura 1. Histograma con curva normal para el rendimiento académico.

Clima social familiar. La dimensión de relaciones está determinada por tres indicadores: cohesión, expresividad y conflictos.

La cohesión (CO) mide el grado en el que los miembros del grupo familiar están compenetrados y se apoyan entre sí. Para el análisis se utilizaron seis elementos para determinar un coeficiente de confiabilidad alfa de Cronbach de .779 y una media de $4.22(D E=.700)$.

La expresividad (EX) explora el grado en el que se permite y anima a los miembros de la familia a actuar libremente y a expresar directamente sus sentimientos. Se utilizaron cuatro elementos con un coeficiente de confiabilidad alfa de Cronbach de .553 y una media de $3.19(D E=.822)$.

El indicador de conflictos (CT) es el grado en el que se expresan libre y abiertamente la cólera, la agresividad y el conflicto entre los miembros de la familia. Se utilizaron cinco elementos con un coeficiente de confiabilidad alfa de Cronbach de .550 y una media de 1.68 $(D E=.631)$.

La dimensión de desarrollo evalúa la importancia que tienen dentro de la familia ciertos procesos de desarrollo personal que pueden ser fomentados o no por la vida en común. Esta dimensión está integrada por las siguientes áreas: autonomía actuación, intelectual-cultural, social-recreativa y moralidad-religiosidad.

El área de autonomía (AU) mide el grado en el que los miembros de la familia están seguros de sí mismos, son independientes y toman sus propias decisiones. En el análisis se utilizaron cinco elementos con un coeficiente de confiabilidad alfa de Cronbach de .527 y una media de $2.61(D E=.790)$.

El área de actuación (AC): observa el grado en el que las actividades (tales como el colegio o el trabajo) se enmarcan en una estructura orientada a la acción-competencia. En el análisis 
se utilizaron seis elementos con un coeficiente de confiabilidad en el alfa de Cronbach de .545 y una media de 3.40 $(D E=.696)$.

El área intelectual-cultural (IC): investiga el grado de interés en las actividades de tipo político, intelectual, cultural y social. En el análisis se utilizaron cinco ítems con un coeficiente de confiabilidad alfa de Cronbach de .594 y una media de $3.32(D E=.797)$.

$\mathrm{El}$ área social-recreativa (SR) explora el grado de participación en actividades de esparcimiento. En el análisis se utilizaron cinco elementos con un coeficiente de confiabilidad alfa de Cronbach de .569 y una media de 3.07 ( $D E=.735)$.

El área de moralidad-religiosidad (MR) permite observar la importancia que se le da a las prácticas y valores de tipo ético y religioso. En el análisis se utilizaron cinco elementos con un coeficiente de confiabilidad alfa de Cronbach de .657 y una media de $3.51(D E=.841)$.

Por otro lado, la dimensión de estabilidad proporciona información sobre la estructura y organización de la familia y sobre el grado de control que normalmente ejercen unos miembros de la familia sobre otros, integrada por las siguientes áreas: organización y control.

El área de organización (OR) indaga sobre la importancia que se le da en el hogar a una clara organización y estructura al planificar las actividades y responsabilidades de la familia. En el análisis se utilizaron seis elementos para determinar un coeficiente de confiabilidad alfa de Cronbach de .627 y una media de $4.02(D E=.648)$.

$\mathrm{El}$ área de control $(\mathrm{CN})$ explora el grado en el que la dirección de la vida familiar se atiene a reglas y procedimientos establecidos. En el análisis se utilizaron cuatro elementos con un coeficiente de confiabilidad alfa de Cronbach de .607 y una media de $3.18(D E=.844)$.

Al realizar una descripción general del clima social familiar, se encontró que el promedio más alto en las áreas del clima social familiar se presenta en cohesión $(M=4.22, D E=.700)$ y el menor promedio en conflictos $(M=1.68$, $D E=.631$ ). Además, se observó que la subescala de control presenta una mayor desviación $(D E=.844)$ con respecto al promedio, indicando mayor variabilidad en las respuestas de los sujetos.

\section{Análisis de los ítems por dimen-} sión. La dimensión relaciones está compuesta por las escalas cohesión, expresividad y conflicto. Obtuvo una media de 3.98 y una desviación estándar de .515 . De los 15 ítems que forman la dimensión de relaciones, los dos con mayor valoración en sus medias corresponden a la dimensión de conflicto, los cuales se interpretan a la inversa; es decir, los estudiantes afirman que no se molestan al grado de golpear o romper algo ni se critican frecuentemente; sin embargo, dicen estar fuertemente unidos y ayudarse unos a otros. Por el contrario, los ítems que obtuvieron las medias más bajas y que indican una menor ocurrencia tienen que ver con hablar abiertamente de lo que quieren o les parece, comentar sus problemas y platicar sobre el manejo de las finanzas en el hogar.

La dimensión desarrollo está compuesta por las escalas autonomía, actuación, intelectual-cultural, social-recreativo y moralidad-religiosidad. Obtuvo una media de 3.19 y una desviación estándar de .516. De los 26 ítems que forman la dimensión desarrollo, en los tres con mayor valoración, los estudiantes comentan que se esfuerzan por hacer las cosas cada vez mejor y que el vínculo familiar es muy importante para ellos 


\section{CLIMA SOCIAL FAMILIAR Y RENDIMIENTO ACADÉMICO}

porque aprenden algo nuevo o diferente y porque la familia es vital para triunfar en la vida. Por el contrario, los ítems que obtuvieron las medias más bajas se relacionan con los espacios en la familia para conversar sobre temas políticos y sociales, la competencia entre los miembros de la familia con la consigna de que gane el mejor y la asistencia en familia a cursos de capacitación por afición o interés.

La dimensión estabilidad está compuesta por las escalas organización y control. Obtuvo una media de 3.69 y una desviación estándar de .634. De los 10 ítems que forman la dimensión estabilidad, en los tres con mayor valoración en sus medias los estudiantes comentan que en sus casas sus dormitorios quedan limpios y ordenados, al igual que el resto de la casa y que colaboran recogiendo los servicios de cocina y otros inmediatamente después de comer. De la misma forma, en los ítems que se obtuvieron las medias más bajas tienen que ver con la importancia que se le da al cumplimiento de las normas, la rigidez y obligatoriedad en el cumplimiento de estas y las decisiones que se deben tomar en casa solamente en manos de una persona.

\section{Análisis correlacional}

Se observó una correlación positiva significativa entre el clima social familiar y el rendimiento académico $(r$ $=.227, p=.001)$. Cuanto mejor clima familiar reportó percibir el estudiante, mejor resultó su rendimiento académico, y a la inversa. Hay que considerar, sin embargo, que la relación es relativamente débil, ya que una variable solo explica el $5 \%$ de la varianza de la otra. En la Figura 2 se puede observar la fuerza de la relación, ya que los datos tienden a formar un círculo más que una elipse alargada en diagonal.
Con la intención de tener mayor información con respecto a la relación entre las variables, se realizó la prueba estadística considerando las dimensiones del clima familiar. Se encontró que el rendimiento correlacionó más fuertemente con la dimensión de relaciones $(r=.247, p=.000)$, seguida por la estabilidad $(r=.236, p=$ .001) y, por último, por la dimensión de desarrollo $(r=.146, p=.037)$.

Al observar diferencias en el clima social familiar según el género del estudiante, se encontró que únicamente existe diferencia significativa en dos de las subescalas: en el área de la actuación $\left(t_{(202)}=2.243, p=.026\right)$, de tal forma que los hombres $(M=3.5, D E=0.742)$ mostraron una media significativamente mayor que la de las mujeres $(M=3.3$, $D E=0.632)$, y en el área de control $\left(t_{(202)}\right.$ $=2.514, p=.013)$, donde igualmente los hombres $(M=3.3, D E=0.824)$ obtuvieron una media más alta que las mujeres $(M=3.0, D E=0.842)$. Al considerar las dimensiones según el género, únicamente se encontró diferencia en la estabilidad $\left(t_{(202)}=2.228, p=.027\right)$, de tal forma que los hombres $(M=3.8, D E=0.617)$ perciben mayor estabilidad que las mujeres $(M=3.6, D E=0.645)$.

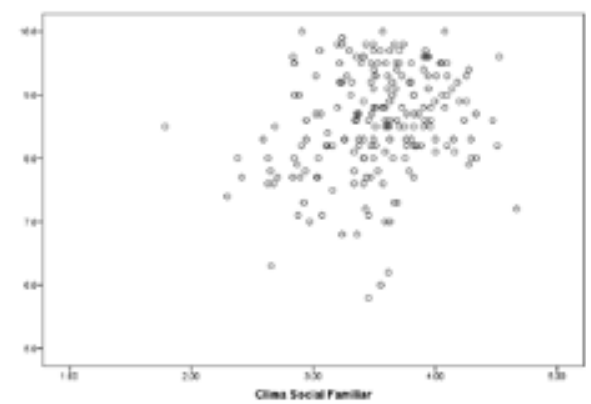

Figura 2. Diagrama de dispersión entre el clima social familiar y el promedio del estudiante. 


\section{Discusión}

Los resultados obtenidos mostraron una relación positiva entre el clima social familiar y el rendimiento académico de los alumnos particpantes. Los resultados coinciden con los de otras investigaciones (Garcés Palacios, 2017; Gonzales Pajuelo y Pereda Infantes, 2009; Guerrero Narbajo, 2014; Pérez Flores, 2015; Pretel Bacilio y Soto Gamboa, 2014; Retamozo González, 2012; Tapia de los Santos, 2014), quienes afirman que la relación entre el clima social familiar y el rendimiento académico son significativos. Según Razeto (2016), los padres con mayor nivel educativo presentan un mayor compromiso con la educación de sus hijos desde la casa. La manera en que los padres organizan a sus familias tiene una gran incidencia sobre el rendimiento escolar de sus hijos.

Por otro lado, los resultados obtenidos en esta investigación contradicen lo manifestado por Hernández Pérez (2015) y Espinoza Heredia y Vera Calderón (2017), quienes concluyen que el bajo rendimiento académico escolar que muestran los alumnos no tiene ninguna relación con el ambiente familiar en que viven los estudiantes.

Se encontró, además, que la relación del rendimiento académico de los estudiantes es más fuerte con la dimensión de relaciones. Es interesante, ya que esta dimensión debería fortalecer el clima social familiar desde la perspectiva de la libre expresión, el apoyarse unos a otros, la compenetración, el actuar libremente, el poder expresar directamente lo que sienten, el grado de comunicación, así como el conflicto que se puede presentar entre los miembros de la familia. Es probable que algunos de los padres estén ayudando a sus hijos con los deberes escolares, aunque en estudio no se pudo corroborar en qué medida. En suma, la relación entre las variables es baja, ya que una variable solo explica el $5 \%$ de la varianza de la otra.

Las dimensiones relaciones y estabilidad son las que más se relacionaron con el rendimiento académico. Si la relación y la estabilidad de las familias es positiva, esto ayuda positivamente al rendimiento académico de los niños. Lo que favorece un clima social familiar, de acuerdo con los resultados encontrados, no son las actividades de superación personal, el divertirse o recrearse, las actividades de origen intelectual-cultural, como el aprender a tocar un instrumento, disfrutar de una interesante lectura o el apreciar una obra de arte. Tampoco las actividades de origen social-recreativo, como el disfrutar de los amigos, las excursiones y los paseos y mucho menos lo que se relaciona directamente con lo moralidad-religiosidad. Precisamente, de las tres dimensiones de esta investigación, la de desarrollo fue la que mostró relaciones menos significativas con el rendimiento académico.

No es la competencia en actividades de esparcimiento, las prácticas religiosas ni las actividades de tipo intelectual, cultural o social las que fortalecen significativamente el clima social familiar en esta investigación, sino el grado de organización, comunicación e interacción que poseen los niños y sus progenitores sobre lo que viven y sienten, el apoyo mutuo, cuando no se les permite hacer lo que quieren y cuando hay cohesión y no hay conflictos en el círculo familiar. El apoyo y la organización que haya en el hogar es de suma importancia para los jovencitos; eso establece la seguridad que muestran en la ejecución de sus deberes escolares. 


\section{CLIMA SOCIAL FAMILIAR Y RENDIMIENTO ACADÉMICO}

El involucramiento de los padres en la educación de sus hijos está asociado positivamente con el desempeño escolar tanto de niños como de niñas. Es de suma importancia que la familia intervenga de manera positiva en el desarrollo cognitivo, psicológico y social de cada niño (Razeto, 2016). La escuela sirve de apoyo para la educación que debe iniciar en el hogar. Es un error pensar que la mayor responsabilidad la tienen los maestros en las escuelas (White, 2010).

\section{Referencias}

Anabalón Mercado, M., Carrasco Paiva, S., Díaz Elgueta, D., Gallardo Urrutia, C., y Cárcamo Vásquez, H. (2008). El compromiso familiar frente al desempeño escolar de niños y niñas de educación general básica en la ciudad de Chillán. Horizontes Educacionales, 13(1), 11-21.

De la Torre Holguin, J. G. (2015). Clima familiar y elección vocacional en estudiantes del quinto grado de educación secundaria de la I. E. $N^{o}$ 81007 “Modelo"-Trujillo-2015 (Tesis doctoral). Universidad Privada Antenor Orrego, Trujillo, Perú.

Diario Oficial de la Federación. (2013). Acuerdo número 696 por el que se establecen normas generales para la evaluación, acreditación, promoción y certificación en la educación básica. Recuperado de http://www.dof.gob.mx/ nota_detalle.php?codigo $=5314831 \&$ fecha $=20 /$ $09 / 2 \overline{0} 13$

Edel Navarro, R. (2003). El rendimiento académico: concepto, investigación y desarrollo. Revista Electrónica Iberoamericana sobre Calidad, Eficacia y Cambio en Educación, 1(2). Recuperado de https://revistas.uam.es/index.php/ reice/article/view/5354/5793

Egremy, N. (2010). La educación en México, zona de desastre: OCDE. Revista Contralínea, 167. Recuperado de https://www.contralinea.com .mx/archivo-revista/2010/01/31/la-educacionen-mexico-zona-de-desastre-ocde/

Espinoza Heredia, A. y Vera Calderón, L. (2017). Clima social familiar y rendimiento académico en los estudiantes de la Escuela Profesional de Economía de la Universidad Nacional de San Martín Tarapoto, 2016 (Tesis de licenciatura). Universidad Peruana Unión, Tarapoto, Perú.

Garcés Palacios, L. (2017). Relación entre el clima social familiar y el rendimiento académico del alumnado de primero y segundo grado de secundaria de la I. E. particular Virgen de la Puerta (Tesis de licenciatura). Universidad Católica Los Ángeles Chimbote, Perú.

Gerstenfeld, P. (1995). Comparación regional del impacto de las características del hogar en el logro escolar. En P. Gerstenfeld, A. Franssen, A. Salinas, A. M. Cerda, V. Edwards y M. V. Gómez (Eds.), Variables extrapedagógicas y equidad en la educación media: hogar, subjetividad y cultura escolar (pp. 13-37). Santiago de Chile: Naciones Unidas, Comisión Económica para América Latina y el Caribe.

Gil Flores, J. (2009). Hábitos y actitudes de las familias hacia la lectura y competencias básicas del alumnado. Revista de Educación, 350, 301-322.

Gonzales Pajuelo, O. A. y Pereda Infantes, A. (2009). Relación entre el clima social familiar y el rendimiento escolar de los alumnos de la institución educativa No 86502 "San Santiago" de Pamparomás en el año 2006, Perú (Tesis de licenciatura). Universidad César Vallejo, Chimbote, Perú.

González Barbera, C. (2003). Factores determinantes del bajo rendimiento académico en educación secundaria (Tesis doctoral). Universidad Complutense de Madrid, Madrid, España.

Guerrero Narvajo, Y. G. (2014). Clima social familiar, inteligencia emocional y rendimiento académico de los alumnos de quinto de secundaria de las instituciones educativas públicas de Ventanilla (Tesis de maestría). Universidad Nacional Mayor de San Carlos, Lima, Perú.

Hernández Pérez, G. (2015). Clima social familiar $y$ rendimiento académico en el Colegio Adventista Libertad de Bucaramanga, Colombia (Tesis de maestría). Universidad de Montemorelos, Montemorelos, México.

Holahan, C. J. (1996). Psicología ambiental: un enfoque general. México: Limusa.

Lastre, K., López, L. y Alcázar, C. (2018). Relación entre apoyo familiar y el rendimiento académico en estudiantes colombianos de educación primaria. Psicogente, 21(39), 102-115. https:// doi.org/10.17081/psico.21.39.2825

Lévy-Leboyer, C. (1985). Psicología y medio ambiente. Madrid: Morata.

López Mero, P., Barreto Pico, A., Mendoza Rodríguez, E. R. y del Salto Bello, M. W. A. (2015). Bajo rendimiento académico en estudiantes y disfuncionalidad familiar. MEDISAN: Revista Médica de Santiago de Cuba, 19(9), 91-94.

Lozano Díaz, A. (2003). Relaciones entre el clima familiar y el fracaso escolar en la educación secundaria. Electronic Journal of Research in Educational Psychology, 1(1), 43-66. Recuperado de http://www.romsur.com/educa/ fracasoesco.htm 


\section{SOTO OVARES Y RODRÍGUEZ GÓMEZ}

Martínez González, A., Inglés Saura, C. J., Piqueras Rodríguez, J. A. y Ramos Linares, V. (2010). Importancia de los amigos y los padres en la salud y el rendimiento escolar. Electronic Journal of Research in Educational Psychology, 8(1), 111-138. Recuperado de http://repositorio.ual .es/bitstream/handle/10835/793/Art_20_372 .pdf?sequence $=1$

Mikulic, I. M. y Casullo, G. L. (2016). Algunas consideraciones acerca del concepto de clima social y su evaluación. Recuperado de http:// www. bibliopsi.org/ docs/carreras/obligatorias/ CFG/psicometricas/mikulic/FICHA \%204.pdf

Moos, R. H., Moos, B. S. y Trickett, E. J. (1989). Escala de Clima Social, familia, trabajo, instituciones penitenciarias, centro escolar ( $3^{\text {ra }}$ ed.). Madrid: TEA.

Moreno Ruiz, D., Estévez López, E., Murgui Pérez, S. y Musitu Ochoa, G. (2009). Relación entre el clima familiar y el clima escolar: el rol de la empatía, la actitud hacia la autoridad y la conducta violenta en la adolescencia. International Journal of Psychology and Psychological Therapy, 9(1), 123-136.

Paz, S. (2007). Problemas en el desempeño escolar y su relación con el funcionalismo familiar en alumnos de EGB 1. Revista de la Facultad de Medicina, 8(1), 27-32.

Pérez Flores, M. G. (2015). Modelo explicativo del rendimiento escolar de los alumnos de quinto y sexto grados de educación primaria a partir de sus niveles de ocupación doméstica, actitud hacia el trabajo doméstico y clima familiar (Tesis doctoral). Universidad de Montemorelos, Montemorelos, México.

Pretel Bacilio, T. D. y Soto Gamboa, L. (2014). Relación entre el clima social familiar y el rendimiento académico en estudiantes de segundo de secundaria de la institución educativa Felipe Huamán Poma de Ayala, Ugel 06, Lurigancho Chosica, 2014 (Tesis de licenciatura). Universidad de Enrique Guzmán y Valle, Lima, Perú.

Razeto, A. (2016). El involucramiento de las familias en la educación de los niños: cuatro reflexiones para fortalecer la relación entre familias y escuelas. Revista Páginas de Educación, 9(2),184-201. https://doi.org/10.22235/ pe.v9i2.1298

Retamozo González, R. M. (2012). Clima social familiar y rendimiento académico en estudiantes de educación básica regular (Tesis de licenciatura). Universidad Católica de Santa María, Arequipa, Perú.
Robledo Ramón, P. y García Sánchez, J. N. (2009). El entorno familiar y su influencia en el rendimiento académico de los alumnos con dificultades de aprendizaje: revisión de estudios empíricos. Aula Abierta, 37(1), 117-128.

Roel Favela, A. (2003). Avances en el conocimiento $\mathrm{y}$ tratamiento del bajo rendimiento escolar. $R e$ vista Salud Pública y Nutrición, 4(2). Recuperado de http://respyn2.uanl.mx/iv/2/invitado/ index.html

Rosales Piña, C. R. y Espinosa Salcido, M. R. (2008). La percepción del clima familiar en adolescentes miembros de diferentes tipos de familias. Psicología y Ciencia Social, 10(1-2), 64-71.

Salazar Flores, N., López Sánchez, L. y Romero Ramírez, M. A. (2010). Influencia familiar en el rendimiento escolar en niños de primaria. Universidad Autónoma del Estado de Hidalgo. Revista Científica Electrónica de Psicología ICSa-UAEH, 9, 137-166. Recuperado de https://es.calameo.com/read/ 0040068289a17426a1fc5

Santín González, D. (2007). Influencia de los factores socieconómicos en el rendimiento escolar internacional: hacia la igualdad de oportunidades educativas (Tesis doctoral). Universidad Complutense de Madrid, Madrid, España.

Tapia de los Santos, L. (2014). Clima social familiar y rendimiento académico en estudiantes de quinto de secundaria de la Institución Educativa "Javier Heraud" (Tesis de maestría). Universidad César Vallejo, Trujillo, Perú.

UNESCO. (2000). Educación para todos: situación y tendencia 2000. Evaluación del aprovechamiento escolar. París: Autor.

Vallejo Casarín, A. y Mazadiego Infante, T. J. (2006). Familia y rendimiento académico. $R e$ vista de Educación y Desarrollo, 3(5), 55-59.

White, E. (2010). Conducción del niño. Miami. APIA.

Zavala García, G. W. (2001). El clima familiar y su relación con los intereses vocacionales y los tipos caracterológicos de los alumnos del 5 to. año de secundaria de los colegios nacionales del Distrito del Rímac (Tesis de licenciatura). Universidad Nacional Mayor de San Carlos, Lima, Perú.

\footnotetext{
Recibido: 4 de febrero de 2019 Revisado: 25 de marzo de 2019 Aceptado: 8 de abril de 2019
} 